\title{
Le récit de rêve en Asie orientale : langues et
} genres

Dream Narrative in East Asia: Languages and Genres

Vincent Durand-Dastès et Rainier Lanselle

\section{OpenEdition}

1 Journals

Édition électronique

URL : https://journals.openedition.org/extremeorient/757

DOI : 10.4000/extremeorient.757

ISBN : 978-2-84292-990-9

ISSN : 2108-7105

Éditeur

Presses universitaires de Vincennes

Édition imprimée

Date de publication : 1 décembre 2018

Pagination : $5-14$

ISBN : 978-2-84292-988-6

ISSN : 0754-5010

\section{Référence électronique}

Vincent Durand-Dastès et Rainier Lanselle, "Le récit de rêve en Asie orientale : langues et genres », Extrême-Orient Extrême-Occident [En ligne], 42 | 2018, mis en ligne le 01 décembre 2018, consulté le 08 janvier 2022. URL : http://journals.openedition.org/extremeorient/757 ; DOI : https://doi.org/10.4000/ extremeorient.757 


\title{
Le récit de rêve en Asie orientale : langues et genres
}

\author{
Vincent Durand-Dastès et Rainier Lanselle
}

Le récit de rêve en Asie orientale, c'est d'abord une éblouissante tradition mantique. Les songes marqueurs du destin, déjà enchâssés dans la littérature historiographique chinoise de l'Antiquité pré-impériale ${ }^{1}$, fournirent au fil des siècles la matière de maints recueils spécialisés, depuis les listes laconiques expliquant le sens des figures rencontrées en rêve jusqu'aux véritables sommes oniromantiques rassemblant, organisant et commentant les centaines de rêves glanés dans les sources écrites ${ }^{2}$. La " Clef des rêves du Duc de Zhou » (Zhougong jiemeng), un apocryphe sans cesse réédité jusqu'à nos jours ${ }^{3}$, compte sans doute parmi les clefs des songes les plus diffusées de l'histoire.

Les métaphores des textes taoïstes anciens, au premier rang desquels le Zhuangzi, firent du rêve une figure clef du discours sur la relativité de l'expérience sensible ; le bouddhisme introduisit et entretint à son tour un riche discours sur les songes ${ }^{4}$; enfin, par une sorte de variation confucéenne sur un thème taoïste, bien des penseurs lettrés se demandèrent si le fait même de rêver était compatible avec les progrès apportés par la culture de soi, comme en témoigne la curieuse formulation selon laquelle «Le saint ne rêve pas ${ }^{5}$ ». Métaphores de l'existence, indices de l'avenir, les rêves furent aussi lus comme des symptômes par les traités de médecine chinoise. Les maîtres de

1. Le Zuozhuan comporte ainsi plus d'une trentaine de récits de rêves. $C f$. Strassberg 2008 : 6-7.

2. Sur la littérature oniromantique, les études les plus notables sont celles de Soymié 1959, Lackner 1985, Ong 1985, Eggert 1992, Drettas 2007, Strassberg 2008, Vance 2012. Les meilleures études chinoises sur la « culture chinoise des rêves » lui font aussi une très large place : Liu et Cao 2003, et Fu 1993a, 1993b, 1993c.

3. Drège 1981, Drège et Drettas 2003. Voir aussi Zheng 2005.

4. Pour les rêves dans les discours religieux bouddhistes et taoïstes, voir Strickmann 1988 et 1996. Voir aussi Lin 1995.

5. Diény 2001. 
rituels exorcistes surent y relever la trace des démons à la poursuite desquels ils s'étaient lancés ${ }^{6}$.

Mais ce ne sont pas de ces « rêves savants » que nous avons choisi de nous occuper à travers cette livraison d'Extrême-Orient Extrême-Occident consacrée au sujet. Bien des études leur ont été dédiées, dont on trouvera les références dans la bibliographie de cette introduction. Notre perspective sera avant tout, même si non exclusivement, littéraire ${ }^{7}$. Quels sont les traits rhétoriques du récit de rêve en Asie orientale ? Quel rôle jouent l'image onirique, le récit de rêve, au sein d'un poème, d'une anecdote ou d'un conte classique, d'une pièce de théâtre ou d'un roman long aux maints rebondissements ? Quels rôles, également, jouent les usages langagiers qui servent de support à ces récits ? Quelle langue, autrement dit, parlent les rêves ?

\section{La vie est un songe : les rêves dans la littérature de récit}

Si on les recherche dans les recueils rassemblant les œuvres complètes d'un lettré, on rencontrera en abondance des pièces classiques brèves répondant à des titres tels que «mémoire d'un rêve » (jimeng), « notes sur un rêve » (jimeng), « récit d'un rêve » (shumeng), qu'il s'agisse de brèves notes en prose ou de poèmes. Mais les récits de rêve de la littérature ne prennent tout leur sens que lorsqu'ils s'enchaînent à de plus grands récits, ou s'y insèrent. C'est à ce type de texte que la plupart des études de ce numéro vont s'attacher.

Les premiers textes combinant récits de rêve et ampleur narrative conséquente apparaissent vers le $\mathrm{IX}^{\mathrm{e}}$ siècle avec la naissance de la nouvelle en prose classique chuanqi. Trois textes de ce nouveau genre sont restés célèbres pour avoir illustré le motif de « la vie est un songe ». Il s'agit du « Rêve du millet jaune » (titre original Zhen zhong ji, « Mémoire de l'intérieur d'un oreiller »), de la «Biographie du gouverneur de Nanke »(Nanke taishou zhuan), et de Yingtao qingyi «La Servante Cerise ». Beaucoup plus tard, Pu Songling (16401715), Yuan Mei (1716-1798) ${ }^{8}$ et Ji Yun (1724-1805), les derniers maîtres de ce genre dans son dernier avatar, feront également une large place aux rêves.

6. Sur l'usage des rêves dans la médecine et l'exorcisme, voir par exemple Harper 1987 et 1988, Chen 2011.

7. Les études sur les rêves dans la littérature chinoise sont relativement moins nombreuses. Voir Hales 1976, Hegel 1988, Lin 1992, Zeitlin 1993. La première étude systématique sur la littérature de rêves des Qing (milieu XVII'-fin XVIII ${ }^{\mathrm{e}}$ siècle) est Lucas 2018.

8. Cent trente cinq récits de rêve du recueil de Yuan Mei, « Ce dont le maître ne parlait pas » ont été traduits par Diény et al., 2011. L'article d'Aude Lucas dans ce volume est 
À leur époque toutefois, la langue classique n'est plus seule à porter le récit de rêve : romans et pièces, parfois courtes mais aussi souvent très longues, ont fait leur apparition et occupent une part importante de la scène littéraire depuis la dynastie des Yuan. Ces genres nouveaux eurent tôt fait de réinvestir le thème de la vie comme songe, le redéployant à une échelle encore inédite.

À l'âge du roman en langue vulgaire (tongsu xiaoshuo), le mot de « rêve » apparaît bien souvent dans les titres des ouvres : si la plupart des romans sont en effet qualifiés de $j i$ (notes, mémoires), zhuan (biographies, traditions) ou yanyi, « développement et explication du sens de... », un nombre significatif de titres de récits en langue vulgaire s'achèvent par le mot de « rêve », donnant à celui-ci une valeur quasi générique. Ce faisant, on suggère au lecteur que le monde dans lequel il va pénétrer en tournant les pages aura la fluidité et la fragilité d'un paysage onirique, comme si le modèle de « la vie est un songe » devenait un cas particulier du récit romanesque, voire pouvait être assimilé au genre dans son entier. L'exemple le plus connu est bien sûr " Le Rêve du pavillon rouge » (Honglou meng), mais de nombreuses autres œuvres parues entre les $\mathrm{XVII}^{\mathrm{e}}$ et $\mathrm{XIX}^{\mathrm{e}}$ siècles affichent elles aussi dans leur titre même la couleur de l'expérience rêvée. Si l'on trouve dans ces « romans en forme de rêve » une prédominance d'histoires d'amours ou tout au moins de romans de mœurs, d'autres évoquent le destin de personnages historiques controversés, dont la grandeur et la chute ont laissé dans l'histoire comme le souvenir d'un rêve ou... d'un cauchemar.

\section{Rêver en langue vulgaire}

Si le récit en langue vulgaire chinois s'inscrit de cette façon dans la tradition littéraire onirique classique, par d'autres aspects elle lui tourne résolument le dos : à l'opposé de la concision et de la parataxe, le récit en langue vulgaire, déployé, bavard et bruyant, inaugure un nouvel âge du récit de rêve littéraire. En un sens, le rêve devient plus explicite : l'identité des protagonistes est plus marquée, les enchaînements paraissent moins évanescents ; la temporalité du rêve, son surgissement, son déroulement, son évanouissement final, appellent de nouveaux procédés. Au théâtre, les scènes de rêve imposent aux comédiens

consacré à des récits oniriques de Pu Songling et Yuan Mei ; ils sont traités en détail dans Lucas, 2018. 
qui les jouent une continuité dans le geste et la parole ${ }^{9}$ qui contraignait beaucoup moins le protagoniste d'un récit classique.

En un sens, les rêves des récits en langue vulgaire des Ming et des Qing peuvent être rapprochés du récit de rêve occidental, ou tout au moins de ce que celui-ci deviendra à partir de l'âge romantique : bien des récits de rêve des Ming ou du début du Qing nous frappent par ce que l'on a envie d'appeler leur « modernité ${ }^{10}$ ». Mais les procédés rhétoriques patiemment bâtis par le récit en langue classique ne s'évanouissent pas pour autant : la poésie, les allusions issues de la littérature classique demeurent présentes comme des incrustations au sein du récit en langue vulgaire et apportent à la littérature prémoderne l'héritage d'une culture onirique construite depuis l'Antiquité.

\section{Randonnées à travers l'imaginaire des rêves}

C'est à la traversée de certains des aspects de cette sensibilité est-asiatique que la collection d'articles présentée ici invite. En sollicitant des contributions pour ce numéro thématique, ses éditeurs ont en effet moins visé à documenter un savoir sur les rêves qu'un imaginaire des rêves, tel qu'il s'exprime à travers la littérature.

La frontière entre ce savoir et cet imaginaire est, au vrai, ténue. C'est pourquoi il nous a semblé qu'il fallait partir de ce qui n'avait jamais cessé de nourrir les thématiques littéraires, à savoir le rêve comme vision : vision non pas d'une scène vécue comme imaginaire, mais vécue comme en provenance d'un au-delà.

Brigid E. Vance ouvre le bal par une plongée dans ce qui est et reste une préoccupation majeure de la sphère officielle est-asiatique : la question de la légitimité politique, à laquelle le rêve ne saurait faire moins que de prêter ses visions. Ce sera la seule partie du recueil où il sera question des encyclopédies oniromantiques. Le rêveur est ici princier. Centré sur l'étude du Meng zhan $y i$ zhi («Sens général des rêves et de leur divination », compilé par Chen Shiyuan [1516-1595]), l'article montre toute la batterie d'arguments - de la

9. Voir le témoignage de Sun Yumin (1940-) sur son jeu de la scène du « Rêve insensé » (Chimeng) dans la pièce Lanke shan. Sur les pièces de théâtre à thème onirique : Liao 2000.

10. Cf. Gollut 1993. 
glyphomancie ${ }^{11}$ aux significations symboliques - mis en œuvres à différentes époques pour constituer de véritables archives oniriques ad usum Caesar ${ }^{12}$.

Avec l'article de Marion Eggert, nous sommes conviés à une incursion en Corée, dans le monde des morts et des rêves à l'époque Chosŏn (XVII ${ }^{\mathrm{e}}$ $\mathrm{XIX}^{\mathrm{e}}$ siècles). Les récits de rêves que l'on trouve dans les chemun (jiwen en chinois), dans les oraisons funèbres, sont l'occasion d'un aperçu très intime de l'expression de la subjectivité, celui où s'exprime le rapport à la mort, au deuil, et plus généralement le rapport à l'Autre perdu, qu'il soit objet d'amour ou d'amitié.

Hiroshi Araki propose une étude centrée sur le grand roman classique japonais, le Genji monogatari, le «Roman du Genji ». Sa « note de lecture », richement référencée, étudie une notion qu'il emprunte à la peinture japonaise et chinoise anciennes, celle de fukidashi, ces « bulles » que l'on voit sortir de la tête des rêveurs pour figurer la scène qui leur apparaît dans leur état de sommeil. Cet angle d'attaque permet de poser une question latente chez nombre d'auteurs de toutes époques : qui est le vrai propriétaire du rêve ?

Le deuxième chapitre est dédié à deux formes de rapport entre le rêve et son effet dans l'expression poétique. Dans la première, Stéphane Feuillas nous emmène en compagnie de Su Shi et de ses (quasi-)contemporains, pour étudier l'émergence d'un motif, poétique, celui du mengzhong zuo, du « composé en rêve ». Cette manière d'écrire consiste à extraire une parole d'un rêve sous la forme d'un vers, lequel sert de point de départ à la composition de tout un poème. Association libre en somme, que l'auteur appelle joliment mise en " écrin poétique » de la parole onirique, où sont mis en scène ses effets d'étrangeté. Sous une forme poétique cette fois assumée, le sujet peut donner voix à son chagrin : c'est le cas par exemple du très beau poème composé par Mei Yaochen au sortir d'un rêve où il s'est promené au bord du fleuve avec sa femme morte quatre ans plus tôt. Avec Su Shi, le rêve devient une formule poétique servant à une défense assumée de l'illusion.

Yanning Wang centre son étude sur un genre particulier, celui des youxian $s h i$, des « poésies de randonnées d'immortels », telles que pratiquées dans plus de trois cents œuvres par l'un des poètes majeurs des Qing, Li E. Le genre luimême est très conventionnel, et c'est à travers des allusions permanentes à des états oniriques que l'auteur tout à la fois s'approprie le genre en le rendant plus personnel, et gagne une réputation publique d'esprit de finesse. Il ne fait plus beaucoup de doute que les rêves sont vus comme des formations personnelles,

11. Cf. Strassberg 2014.

12. Voir également Vance 2017. 
et le rêveur veut désormais des rêves qui servent ses désirs - dont celui d'atteindre l'immortalité, mais cette fois littéraire.

C'est à la psychanalyse qu'est empruntée la notion d' « autre scène » (der andere Schauplatz) qui sert de titre à la troisième partie du volume. Plutôt qu'un autre monde, celui des immortels, l'époque prémoderne fait le constat que le monde des rêves relèverait d'une étrangeté intérieure, avec sa dimension sexuelle. C'est ce qu'illustrent les trois derniers articles, centrés sur la littérature de récit, roman, conte et théâtre, allant des Yuan au milieu des Qing. Ces trois articles sont l'avatar d'un panel, qu'avait présidé Keith McMahon, présenté au congrès de l'Association for Asian Studies à Chicago en 2015, conjonction qui est à l'origine de cette livraison de la revue.

Vincent Durand-Dastès centre son analyse sur le Chan zhen yishi, «Histoires oubliées des maîtres de dhyāna et des parfaits », un roman de la fin des Ming. Les scènes oniriques y abondent, et on ne peut s'empêcher d'évoquer leur dimension de parodie. D'un côté, rien ne semble devoir faire davantage justice à la grande tradition oniromantique que bien des interprétations données des rêves des protagonistes ; mais d'un autre, tant de topoï, de lieux communs, par leur accumulation même, trahissent leur vraie destination : donner voix à un désir privé, qui se cache de manière souvent comique sous les oripeaux de la « tradition ». « L'originalité de nos récits », écrit Durand-Dastès, « consiste à mêler ces lieux communs divinatoires à des inventions tout à fait originales, où destinée et hasard se voient habilement mêlés. » Tel personnage, explique-t-il, fait un rêve de mariage avec une belle, « sans avoir consciemment espéré cette annonce heureuse [...] : celle de son désir latent a suffi au dieu entremetteur. »

Avec Aude Lucas, l'analyse se concentre non sur une série de rêves, mais sur deux rêves seulement, tirés de la tradition tardive des contes ou «notes diverses » en langue classique. L'étude d'un récit particulièrement important de Pu Songling, l' «Histoire de Shi Qingxu », où le rêve prend son sens par rapport à toute une mise en abîme qui en forme le contexte, donne l'occasion à Lucas d'interroger un rapport, " moderne », du rêve à la réalité vécue, subjective, ce qu'elle fait en reprenant les notions lacaniennes d'imaginaire, de symbolique et de réel. Le conte est comme un apologue de la valeur du rêve dans la période prémoderne : tout comme le personnage fait un rêve dont il ignore complètement que le contenu est le sien propre, le rêveur, dans un monde où le surnaturel reflue, ne sait plus à qui attribuer ses visions.

Une vision mélancolique du rêve est celle qui s'exprime dans le songe qui vient conclure l'histoire des amours de Zhang Sheng et de Cui Yingying, dans la pièce le Xixiang ji («Le Pavillon de l'ouest »), une œuvre datant des Yuan ( ca 1300), mais dont le destin s'étend sur sept siècles d'histoire littéraire. Dans cet article, Rainier Lanselle entreprend l'analyse d'un seul et unique 
rêve, mais placé à un point stratégique. Il y est question du désir, certes, mais dans ce que le désir a comme fâcheuse tendance - nous dirions structurelle - à rater son objet - et le rêve dès lors à se perpétuer comme une énigme sans véritable réponse. Le rêve retourne ainsi à son insondable premier, cet objet des devins, cet « ombilic » dont Freud disait qu'on ne pouvait rien en dire. Lecture désenchantée du rêve qui étend son ombre mélancolique jusque sur le Rêve du pavillon rouge.

Il ne restera plus au lecteur de bonne volonté qui nous aura suivi jusque-là de lire, pour finir, le passionnant contrepoint que donne Jacqueline Carroy ${ }^{13}$, sur l'imaginaire et les littératures du rêve en Occident. En regardant de l'extérieur, depuis cet Occident marqué par la tradition chrétienne, ces rêves de l'autre bout du continent, elle nous fait mieux sentir ce que peut apporter cette autre volonté, en Chine, en Corée, au Japon, ailleurs, de donner voix au rêve à travers l'écrit. Pour la paraphraser, nous serons tentés de dire en concluant que si l'écriture fait quelque chose aux rêves, il est certain que les rêves font, en Asie orientale, beaucoup de choses à l'écriture.

\section{BIBLIOGRAPHIE}

CARROY Jacqueline (2012). Nuits savantes. Une histoire des rêves (1800-1945). Paris : Éditions de l' EHESS.

CHEN Hsiu-fen (2011). « Dreaming Between Passion and Repression : Medical Views of Demon Dreams, Demonic Fetuses, and Female Sexual Madness in Late Imperial China ». Late Imperial China, 32(1) : 51-82.

DIÉNY Jean-Pierre (2001). «Le Saint ne rêve pas : de Zhuangzi à Michel Jouvet ». Études chinoises, $\mathrm{XX}(1-2): 127-200$.

DRÈGE Jean-Pierre (1981). «Clefs des songes de Touen-huang ». In SoYMIÉ Michel (dir.), Nouvelles contributions aux études de Touen-huang. Genève, Librairie Droz : 205-249.

DRÈGE Jean-Pierre et Dimitri DRETTAS (2003). « Oniromancie ». In KALINOWSKI Marc (dir.), Divination et société dans la Chine médiévale. Étude des manuscrits de Dunhuang de la Bibliothèque nationale de France et de la British Library. Paris, Bibliothèque nationale de France : 369-404.

DRETTAS Dimitri (2007). « Le rêve mis en ordre. Les traités onirologiques des Ming, à l'épreuve des traditions divinatoire, médicale et religieuse du rêve en Chine », thèse de doctorat. Paris, EPHE.

13. Voir Carroy 2012. 
EGGERT Marion (1992). Rede von Traum : Traumaufassungen der Literatenschicht in späten kaiserlichen China. Stuttgart, Franz Steiner.

FU Zhenggu 傅正谷 (1993a). Zhongguo meng wenhua 中國夢文化. Pékin, Zhongguo shehui kexue chubanshe.

FU Zhenggu 傅正谷 (1993b). Zhongguo meng wenhua cidian 中國夢文化詞典. Taiyuan, Shaanxi gaoxiao lianhe chubanshe.

Fu Zhenggu 傅正谷 (1993c). Zhongguo meng wenxue shi : Xian Qin liang Han bufen 中國夢文學史 : 先秦兩漢部分. Pékin, Guangming ribao chubanshe.

Gollut Jean-Daniel (1993). Conter les rêves : la narration de l'expérience onirique dans les auvres de la modernité. Paris, José Corti.

HALES D. R. (1976). «Dreams and the Daemonic in Traditional Chinese Short Stories ». In NiENHAUSER W. H. (dir.), Critical Essays on Chinese Literature. Hong Kong, The Chinese University of Hong Kong : 71-87.

HARPER Donald (1987). «Wang Yen-shou's Nightmare Poem ». Harvard Journal of Asiatic Studies, 47(1) : 239-283.

HARPER Donald (1988). «A note on Nightmare Magic and Ancient and Medieval China ». T'ang studies, $6: 69-76$.

HeGEL Robert (1988). «Heavens and hells in Chinese Fictional Dreams ». In BROWN Carolyn T. (dir.), Psycho-Sinology : The Universe of Dreams in Chinese Culture, 1(10). Boston, University Press of America.

LACKNER Michael (1985). Der chinesische Traumwald. Traditionelle Theorien des Traumes und seiner Deutung im Spiegel der ming-zeitlichen Anthologie Meng-lin hsüan-chieh. Frankfurt/Bern/New York, Peter Lang.

LIAO Tengye 廖藤葉 (2000). Zhongguo mengxi yanjiu 中國夢戲研究. Taipei, Xuesi chubanshe.

LIN Fu-shih (1995). «Religious Taoism and Dreams : An Analysis of the Dream-data Collected in the Yün-ch'i Chi-chien ». Cahiers d'Extrême-Asie, 8 : 95-112.

LIN Shuen-fu (1992). «Chia Pao-yü's First Visit to the Land of Illusion : An Analysis of a Literary Dream in Interdisciplinary Perspective ». CLEAR (Chinese Literature : Essays, Articles, and Reviews), 14 : 77-106.

LIU Wenying 劉文英, CAO, Tianyu 曹田玉 (2003). Meng yu Zhongguo wenhua 夢與 中國文化. Pékin, Renmin chubanshe.

LuCAS Aude (2018). «L'expression subjective dans les récits oniriques de la littérature de fiction des Qing », thèse de doctorat. Paris, Université Sorbonne Paris Cité/ Université Paris-Diderot.

Ong Roberto K. (1985). The Interpretation of Dreams in Ancient China. Bochum, Brockmeyer.

SOYMIÉ Michel (1959). «Les songes et leur interprétation en Chine ». In Sources orientales II, Les Songes et leur interprétation. Paris : Seuil : 275-305.

STRASSBERG Richard E. (2008). Wandering Spirits : Chen Shiyuan Encyclopedia's of Dreams. Berkeley, University of California Press.

STRASSBERG Richard (2014). «Glyphomantic Dream Anecdotes ». In CHEN Jack et David SCHABERG (dir.), Idle Talk : Gossip and Anecdote in Traditional China. Berkeley, University of California Press : 178-193. 
STRICKMANN Michel (1988). «Dreamwork of Psycho-Sinologists : Doctors, Taoists, Monks ». In BROWN Carolyn T. (dir.), Psychosinology : The Universe of Dreams in Chinese Culture. Washington, Woodrow Wilson International Center for Scholars : 25-46.

STRICKMANN Michel (1996). «Les rêves et la divination ». In Mantras et mandarins : le bouddhisme tantrique en Chine. Paris, Gallimard : 291-336.

VANCE Brigid (2012). «Textualizing Dreams in a Late Ming Dream Encyclopedia », thèse de Ph.D. Princeton, Princeton University.

VANCE Brigid E. (2017). « Deciphering Dreams : How Glyphomancy Worked in Late Ming Dream Encyclopedic Divination ». The Chinese Historical Review, 24(1) : 5-20.

YUAN Mei (2011). Ce dont le maître ne parlait pas. DiÉNY Jean-Pierre et al. (éd. et trad). Paris, Gallimard (« Connaissance de l'Orient »).

ZHENG Binglin 鄭炳林 (2005). Dunhuang ben jiemengshu jiaolu yanjiu 敦煌本解夢書 校錄研究. Pékin, Minzu chubanshe.

ZEITLIN Judith (1993). Historian of the Strange : Pu Songling and the Chinese Classical Tale. Stanford, Stanford University Press.

\section{GLOSSAIRE}

Cao Xueqin 曹雪芹

Chan zhen yishi 禪真逸史

chemun 祭文

Chen Shiyuan 陳士元

Chimeng 癡夢

chuanqi 傳奇

Cui Yingying 崔鶯鶯

fanmeng 反夢

fukidashi 吹き出し

Genji monogatari 源氏物語

Honglou meng 紅樓夢

$j i$ 記 (notes, mémoires)

$j i$ 集 (recueils)

Ji Yun 紀昀

jimeng 紀夢 (notes sur un rêve)

jimeng 記夢 (mémoire d'un rêve)

Lanke shan 爛柯山

$\mathrm{Li}$ E 厲鶚

Mei Yaochen 梅堯臣

meng 夢 
Vincent Durand-Dastès et Rainier Lanselle

Meng zhan yi zhi 夢占逸旨

mengzhong zuo 夢中作

Nanke taishou zhuan 南柯太守傳

Pu Songling 蒲松齡

Shi Qingxu 石清虛

shumeng 述夢

Su Shi 蘇軾

Sun Yumin 孙毓敏

tongsu xiaoshuo 通俗小說

xing 醒

Xixiang ji 西廂記

yanyi 演義

Yingtao qingyi 櫻桃青衣

youxian shi 遊仙詩

Yuan Mei 袁枚

Zhang Shanxiang 張善相

Zhen zhong $j i$ 枕中記

Zhougong jiemeng 周公解夢

Zhuangzi 莊子

zhuan 傳

Zi bu $y u$ 子不語 